\title{
Molecular Diagnosis of Fungal Sinusitis Using Limit of Detection
}

\author{
Yaser Ranjibar ${ }^{1}$, Mohammad Hassan Shahhosseiny ${ }^{(i D}{ }^{2}$, Farouq Karimpour ${ }^{1}$ and Fatemeh Keshavarzi \\ (i) $3,{ }^{*}$ \\ ${ }^{1}$ Department of Biology, Kurdistan Science \& Research Branch, Islamic Azad University, Sanandaj, Iran \\ ${ }^{2}$ Department of Microbiology, Shahr-e Ghods Branch, Islamic Azad University, Shahr-e Ghods, Tehran, Iran \\ ${ }^{3}$ Department of Biology, Sanandaj Branch, Islamic Azad University, Sanandaj, Iran \\ "Corresponding author: Department of Biology, Sanandaj Branch, Islamic Azad University, Sanandaj, Iran. Email: gol.keshavarzi@gmail.com
}

Received 2021 April 17; Revised 2021 November 06; Accepted 2021 November 14.

\begin{abstract}
Background: As an inflammatory process that involves the paranasal sinuses, chronic sinusitis (CS) is one of the most prevalent chronic illnesses that affects all age groups. Parasitic fungi are involved in sinusitis infections.

Objective: This study is aimed at the molecular detection of sinusitis caused by such fungi.

Methods: Seventy-two samples were collected from the secretions of maxillary and frontal sinuses of patients from Rasoul-e Akram (PbUH) Hospital in Tehran during sinus operation. Fungal genomic DNA was extracted by a DNP kit. The detection of fungi was carried out by employing a sequence-specific target, namely $m t$ cyte $b$ gene locus, and using primers. Polymerase chain reaction (PCR) was optimized, and the limit of detection (LOD) and specificity tests were performed. The amplicon was cloned by the T/A cloning method, which was used for sequencing and positive control.

Results: The 430-bp PCR product underwent appropriate propagation before being amplified and was observed on $1.5 \%$ electrophoreses gel. The evaluation of the selected primers with seven DNA constructs from another microorganisms demonstrated $100 \%$ specificity. The limit of detection of the optimized test was evaluated up to 50 fungi. Out of 72 samples, $9.7 \%$ were positive for fungi existence.

Conclusions: This study indicated that molecular diagnosis of the target $m t$ cyte $b$ gene using LOD enhances clinical laboratory detection of fungal sinusitis.
\end{abstract}

Keywords: Fungal Sinusitis, $m$ t cyte $b$ Gene, LOD

\section{Background}

The development of fungal colonies in paranasal sinuses in infected or uninfected sinuses is a common complication (1-6). Chronic sinusitis is one of the most prevalent chronic diseases affecting people of all ages (7). In fact, sinusitis is an inflammation of the paranasal sinuses that may be caused by an infection, allergy, or autoimmune diseases (8). Paranasal sinuses consist of four pairs of air-filled cavities at the entrance of the upper airway, which include maxillary, frontal, sphenoid, and ethmoid sinuses (9). In the beginning, sinusitis is caused by a viral infection that usually lasts more than 10 days, and in $99 \%$ of cases, it is completely cured, but in a small number of patients, a secondary bacterial infection may develop, usually caused by aerobic bacteria (10). About $10 \%$ of sinusitis cases requiring surgery are caused by a fungal infection. The infection may be caused by a variety of fungal families. Aspergillus fumigatus is the most common cause of fungal infection of the si- nuses, and the maxillary sinus is the most common site of such fungal infections. The diagnosis of fungal infections of the paranasal sinuses requires sufficient symptoms (4, 5).

Due to the significance of rapid and accurate diagnosis of fungal infections in patients with chronic sinusitis for the timely treatment and prevention of secondary complications, finding a fast, sensitive, easy, low-cost, and available method seems necessary. Another reason for the need for such a method is the time-consuming nature of fungal culture methods. The classic diagnosis of this Fungusis usually made on the basis of microscopic and culture methods. These techniques are time consuming, relatively inaccurate, and require facilities and experience to interpret the results (6). The usual method of laboratory diagnosis of fungi is to cultivate them, which takes a long time (a few days to several weeks) so that their morphological characteristics are definitely indicative of their species (7). In addition, false-negative results are relatively high in 
such cases (8). Numerous studies have been conducted on the diagnosis of chronic sinusitis, and different methods have been used for this purpose. Cultivation, immunological, enzymological, and molecular methods have been proposed, each with its own advantages and disadvantages. Non-cultivation methods differ greatly in terms of velocity, reliability, limit of detection, specificity, accuracy, and costs (6).

Few studies have been conducted on the diagnosis of fungal sinus infections using the PCR method (11-17). In 2003, Biswas performed a molecular analysis of the $m t$ cyte $b$ gene sequence in the fungus Cryptococcus neoformans. In that study, he used the primer pair of E1M4 and rE2M4, which were able to detect the fungus. The study showed a high level of diagnosis and specificity (17).

\section{Objectives}

Therefore, in the present study, the locus $m t$ cytb with high LOD and specificity was used for the rapid diagnosis of fungal isolates in samples of patients with chronic sinusitis admitted to Rasoul-e-Akram (PBUH) Hospital.

\section{Methods}

\subsection{Samples}

Seventy-two samples were collected of maxillary and frontal sinus secretions of patients admitted to Rasool-e Akram (PBUH) Hospital in Tehran for sinus surgery. The samples were collected from patients diagnosed with sinusitis by a hospital Ear, Nose, and Throat specialist while taking into consideration that the disease had reached a chronic stage and the patients had the indications for surgery. Over a nine-month period, 72 specimens were prepared during sinus surgery and poured into vials containing sterile physiological serum.

\subsection{DNA Extraction from Fungi}

In order to optimize the PCR test and place a positive control for the detection of fungi in clinical specimens, Fusarium solani species was cultivated for 48 hours at a temperature of $37^{\circ} \mathrm{C}$ in Sabouraud dextrose agar medium, and from the resulting colonies and the 72 samples, DNA was extracted by the boiling method using a DNP kit (Cinnaclon company) based on the protocol.

\subsection{Optimizing PCR Test}

The primers for the PCR of $m t c y t b$ were E1M4 (Forward: $5^{\prime}$-TGR GGW GCW ACW GTT ATT ACT A-3`) and rE2M4 (Reverse: $5^{\prime}$-GGW ATA GMW SKT AAW AYA GCA TA-3') according to a former study (14). In this method, to optimize the PCR technique, the suitable concentration and volume of the required components of this method were determined, and finally, the required values were obtained in a PCR test in $25-\mu \mathrm{L}$ volume. Also, the temperature profile was optimized by the gradient method to mutually amplify the $m t c y t b$ gene as follows: denaturation at $94^{\circ} \mathrm{C}$ for one minute, annealing at $50^{\circ} \mathrm{C}$ for one minute, and an extension at $72^{\circ} \mathrm{C}$ for two minutes. To obtain better results, we continued the final extension for 10 minutes at the same temperature; this process was performed in 35 cycles. The PCR test was carried out under optimized conditions, and the PCR product was electrophoresed in 1.5\% agarose gel containing SYBER Green in 0.5 TBE X5 buffer.

\subsection{Cloning PCR Products}

After purifying the PCR product, it was cloned using a T/A cloning kit (Fermentas Company) in pTZ57R carrier. The resulting plasmids were extracted by the alkaline lysis method. The PCR method then confirmed the plasmids containing the PCR product (14).

\subsection{Limit of Detection}

Limit of detection (LOD) test was conducted to obtain serial dilutions from fungal DNA. For this purpose, the extracted DNA of the F. solani fungus was used, and its LOD was measured with a nanodrop device and based on the genome copy number formula and the size of the F. solani genome. In this method, the copy numbers of DNA were known, and serial dilution were prepared.

\subsection{Determining PCR Test Specificity}

To determine the specificity, we extracted the DNA of seven common microorganisms in sinusitis, such as Staphylococcus aureus, Streptococcus pneumonia, Escherichia coli, Pseudomonas aeruginosa, and viral species, such as hepatitis B virus, herpes simplex virus, and cytomegalovirus, and performed the PCR test for them with a positive and negative control sample.

\section{Results}

4.1. The Results of Optimization, LOD, and Specificity of PCR

Optimizing the PCR test: After optimizing the PCR test for the concentration of PCR reaction components and 
thermal profile using universal fungi primers, according to the appropriate thermal program, a PCR cycle was performed on it, and fungal DNA was evaluated along with a negative control sample and a marker size on $1.5 \%$ agarose gel. Following the electrophoresis of the PCR product, a band was visualized from the related amplicon with a size of 430 bps (Figure 1). DNA from other fungi, such as Candida albicans, C. neoformans, Aspergillus flavus, Aspergillus parasiticus, and Aspergillus niger, were also used in this experiment, but none of them were amplified with the fE1M4 and rE2M4 primers.

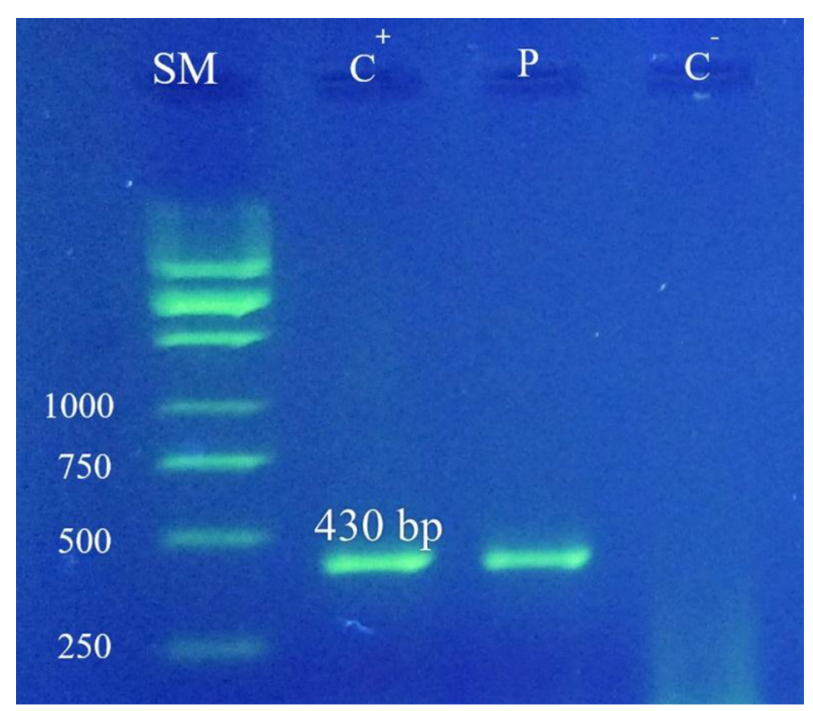

Figure 1. Optimized PCR test for detecting the presence of fungi. SM: DNA marker size DNA Ladder1 kb (Fermentas, Safirazma Company, Iran), $C^{+}$: Positive control, P: PCR product with DNA of Fusarium solani (430 bp), C: Negative control.

Limit of detection: LOD test was conducted to provide serial dilutions from fungal DNA. Limit of detection findings of PCR test revealed that even if the number of fungal DNA amounts to 50, fungal proliferation occurs. When the number is less than 50 , no band could be seen, indicating high LOD of the test (Figure 2).

Polymerase chain reaction test specificity: The PCR specificity test was performed using DNA from seven other common organisms in sinusitis, and no unwanted products or bands were observed (Figure 3).

\subsection{The Results of Performing PCR Test on Patients' Samples}

DNA from 72 samples of paranasal sinuses was extracted by the boiling method using a DNP kit (Cinnaclon Company) and was employed for the PCR test. Finally, 7 (9.7\%) samples were definitely positive (Figure 4).
Figure 5 shows the results of the PCR test in a number of studied samples.

\section{Discussion}

Paranasal sinus diseases account for a significant portion of annual cost of treatment (11). Fungal sinusitis is most commonly found in the age groups of 10 to 60 (15). New approaches to the diagnosis of fungal infections and antifungal therapies have emerged based on recent research into the role of fungi in the development and progression of chronic sinusitis. Wang et al., in 1998 designed a primer of the known cytochrome $b$ amino acid sequence to identify and evaluate the racial evolution of Aspergillus pathogens (10). Walter Buzina examined 12 samples of fungi for the molecular detection of Schizophyllum commune. After culturing the samples, they extracted their DNA and the transcriptional sequence of ribosomal DNA s8 / 5 (5.8s rDNA), and ITS region was amplified by the specific fungal primers ITS4 and ITS5, and the products were sequenced by PCR (12). In 2003, Biswas performed a molecular analysis of the $m t$ cyte $b$ gene sequence in the fungus $C$. neoformans. In that study, he used the primers pair of E1M4 and rE2M4, which were able to detect the fungus. The study had a high level of diagnosis and specificity. In the present study, locus $m t$ cyt $b$ with high a limit of detection and specificity was used for the PCR method. Samples of this study were specifically taken from patients with chronic sinusitis during surgery, and 9.7\% of them were diagnosed with fungi (17).

In Iran, Naghibzadeh et al. reported that through the cultivation method, sinus secretions, and light microscopy of 162 patients with chronic sinusitis, 12 patients were confirmed to have fungal infections, which included 2 (1.2\%) cases of A. flavus, 9 (5.66\%) cases of Alternaria, and 1 (0.6\%) case of Paecilomyces. The prevalence of chronic fungal sinusitis among the population community was about $7.4 \%$, which is lower compared to other causes of sinusitis. According to the studies, the most significant pathogen causing the disease in the family of fungi is Alternaria. Compared to other factors causing sinusitis, fungi are less common (16).

Based on the results of the current study, the evaluation of the selected primers with seven various DNA constructs demonstrated $100 \%$ specificity (Figure 1 ). Also, the LOD of optimized test was evaluated for up to 50 fungi (Figure 2). The PCR specificity test results showed that the PCR test had very high specificity and only reacted toward fungal DNA (Figure 3). In addition, out of 72 samples, 9.7\% 


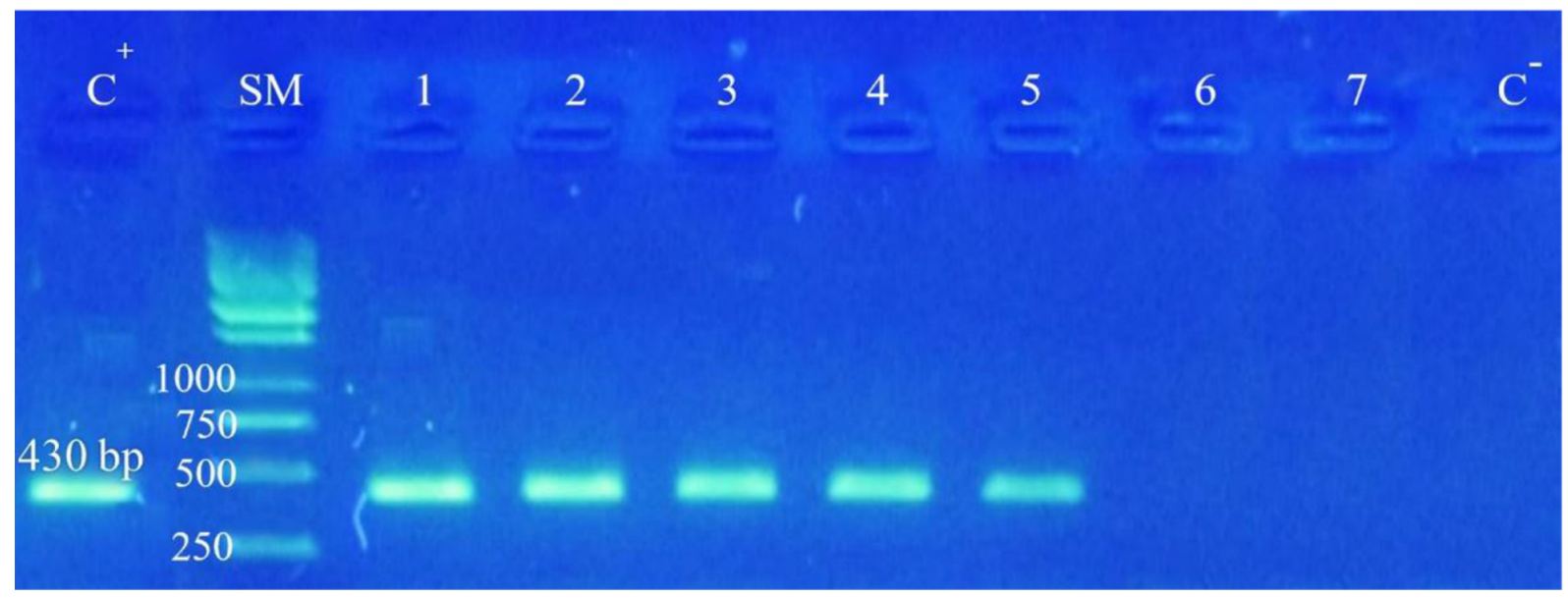

Figure 2. LOD test. C : Negative control, $C^{+}$: Positive control, SM: Size marker kb DNA Ladder 1 (Ferments) 1: 0.1 dilution containing 500 thousand fungal DNA, 2: 0.01 dilution containing 50 thousand fungal DNA, 3: 0.001 dilution containing 5 thousand fungal DNAs, $4: 0.0001$ dilution containing 500 fungal DNAs, 5: $10^{-5}$ dilution 50 fungal DNAs, 6 : $10^{-6}$ dilution, $7: 10^{-7}$ dilution.

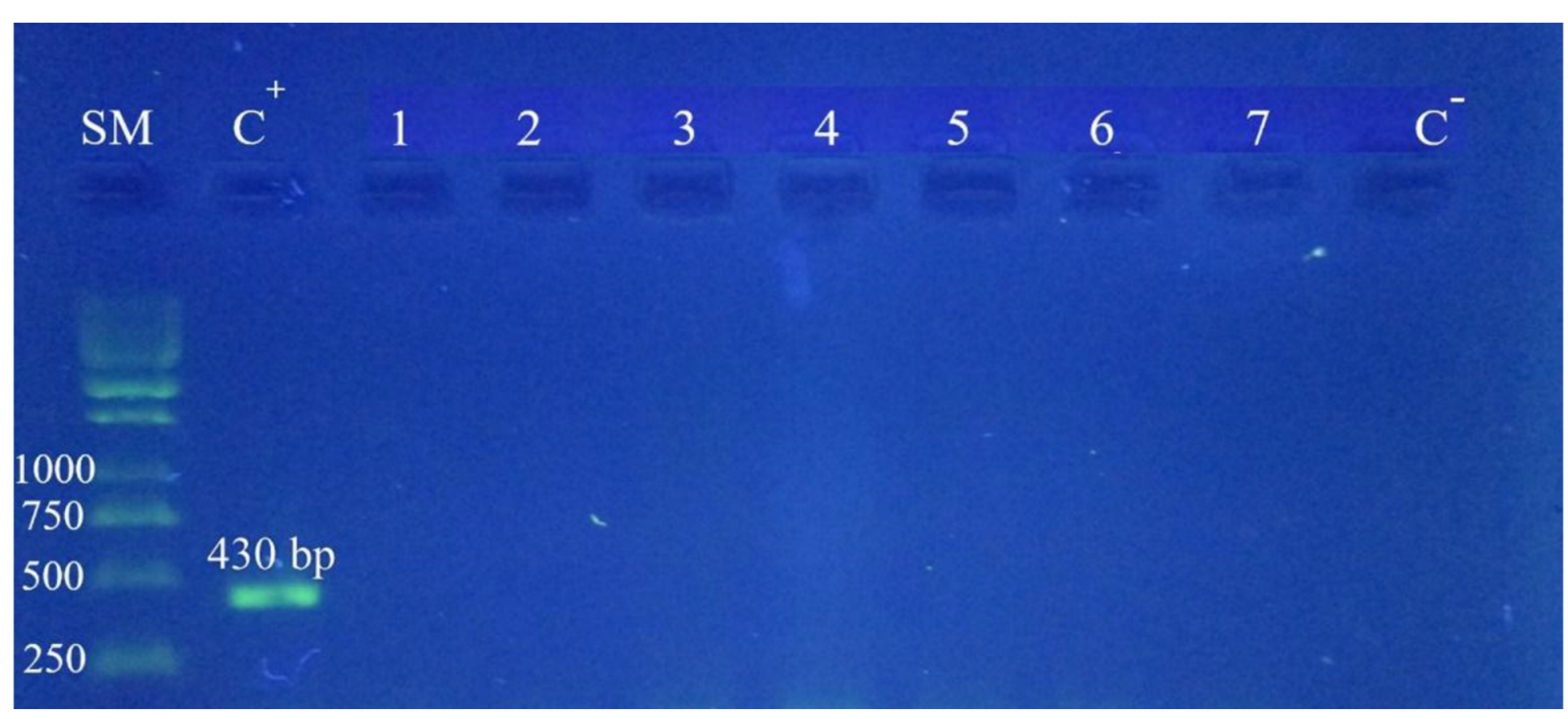

Figure 3. PCR specificity test of Fusarium solani: SM: kb DNA marker size Ladder 1 (Fermentas), $C^{+}$: Positive control sample (F. solani), 1: Staphylococcus aureus, 2: Streptococcus pneumoniae, 3: Pseudomonas aeruginosa, 4: Escherichia coli, 5: Hepatitis B virus, 6: Herpes simplex virus, 7: Cytomegalovirus, C: Negative control.

were positive for fungi existence (Figure 4). Samples of this study were specifically taken from patients with chronic sinusitis during surgery, and 9.7\% of them were diagnosed with fungi.

As mentioned earlier, fungi are one of the contributing factors of chronic sinusitis. The only way to control the disease is early diagnosis. Therefore, the rapid diagnosis of this microorganism can play an essential role in identifying and controlling the pathogen in early stages of the disease (17).
The PCR technique used in this study to detect different types of fungi was highly accurate, and this method can be used to quickly diagnose the organism in patients with sinusitis and other infections caused by the fungal family (17).

One of the limitations of this study was that the high cost did not allow us to use other diagnostic methods such as culture for comparison at the same time and during molecular detection. 


\section{\% of Negative \& Positive Cases}

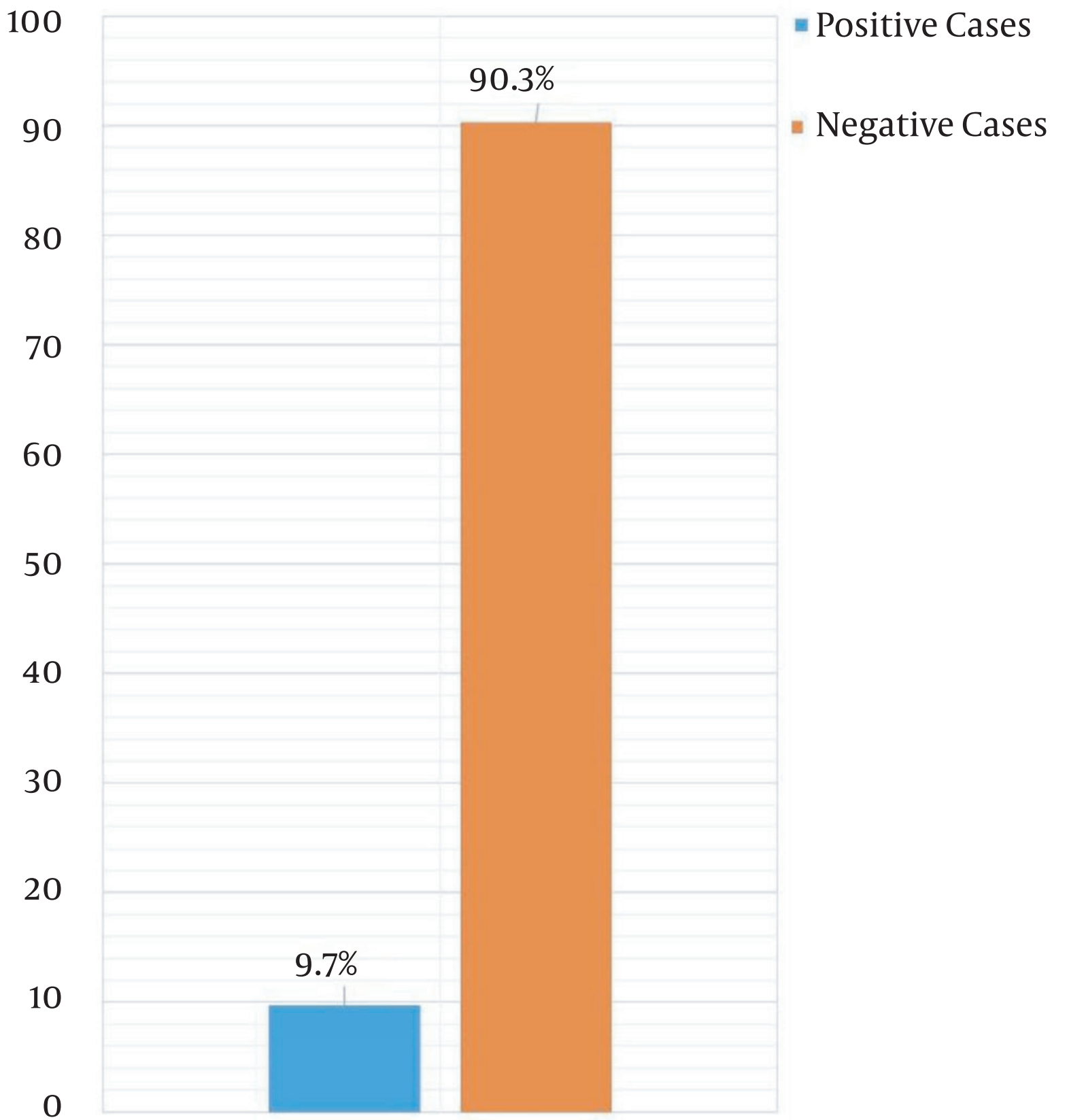

Figure 4. Percentage of positive and negative samples. From 72 samples of paranasal sinuses after DNA extraction PCR, $7(9.7 \%)$ samples were definitely positive, and 65 (90.3\%) were negative.

\subsection{Conclusions}

In the present study, the locus $m t$ cytb was used for the rapid diagnosis of fungal types in samples of patients with chronic sinusitis from Rasoul-e-Akram (PBUH) Hos- pital. Seventy-two samples were collected from the secretions of maxillary and frontal sinuses of patients during sinus operation. After DNA extraction, the detection of fungi was carried out by amplifying the target sequence using 


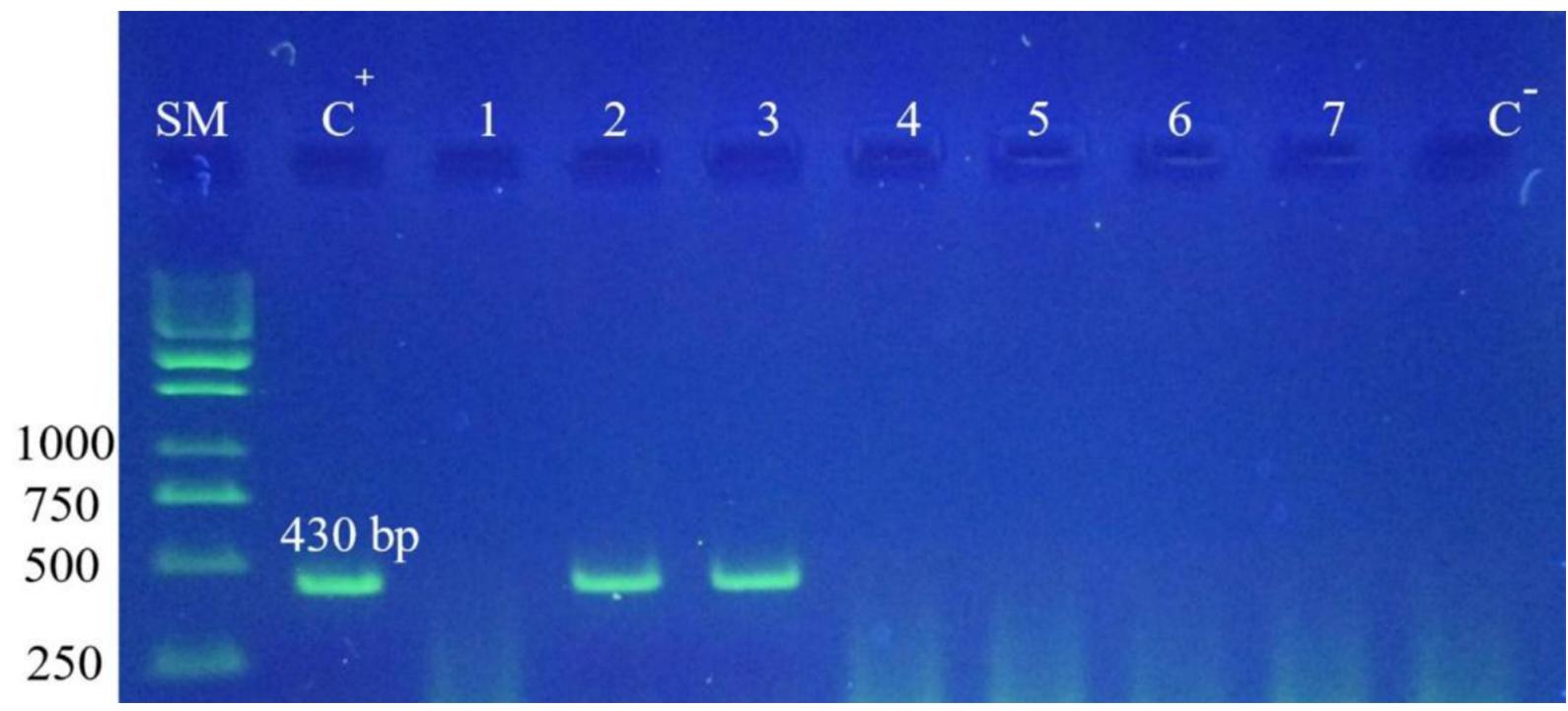

Figure 5. Optimized PCR test to detect the presence of fungus in sinusitis samples. SM: Marker size kb DNA Ladder 1(Fermentas), $\mathrm{C}^{+}$: Positive control, 2 and 3: Positive sample 1 and 7-4: Negative samples $C^{-}$: Negative control.

universal primers. At first, PCR was optimized, and LOD and specificity tests were performed. The amplicon was cloned by the T/A cloning method. Based on the results, the evaluation of the selected primers with seven various DNA constructs demonstrated $100 \%$ specificity. Also, LOD of the optimized test was evaluated up to 50 fungi. Out of the 72 samples, 9.7\% were positive for fungi existence. Samples of this study were specifically taken from patients with chronic sinusitis during surgery, and 9.7\% of them were diagnosed with fungi.

\section{Footnotes}

Authors' Contribution: Fatemeh Keshavarzi: Supervised the study, wrote and revised the manuscript; Mohammad Hassan Shahhosseiny: Supervised the study; Yaser Ranjibar: Performed experiments and analyzed the data; and Farouq Karimpour: Field work and manuscript preparation. All the authors read the paper and approved the final manuscript.

Conflict of Interests: The authors declare that they have no competing interests.

Ethical Approval: The study was ethically approved by the Research Committee of Sanandaj Branch, Islamic Azad University.

Funding/Support: This study was not supported by a grant from funding sources.
Informed Consent: Participants in the research clearly consented to being involved in the study.

\section{References}

1. Brook I. Microbiology of sinusitis. Proc Am Thorac Soc. 2011;8(1):90-100. doi: 10.1513/pats.201006-038RN. [PubMed: 21364226].

2. Vennewald I, Henker M, Klemm E, Seebacher C. Fungal colonization of the paranasal sinuses*. Mycoses. 1999;42:33-6. doi: 10.1111/j.14390507.1999.tb00010.x.

3. Ponikau JU, Sherris DA, Kern EB, Homburger HA, Frigas E, Gaffey TA, et al. The diagnosis and incidence of allergic fungal sinusitis. Mayo Clin Proc. 1999;74(9):877-84. doi: 10.4065/74.9.877. [PubMed: 10488788].

4. Catten MD, Murr AH, Goldstein JA, Mhatre AN, Lalwani AK. Detection of fungi in the nasal mucosa using polymerase chain reaction. Laryngoscope. 2001;111(3):399-403. doi:10.1097/00005537-20010300000006. [PubMed: 11224767].

5. Stringer SP, Ryan MW. Chronic invasive fungal rhinosinusitis. Otolaryngol Clin North Am. 2000;33(2):375-87. doi: 10.1016/s00306665(00)80012-2.

6. Massa HM, Cripps AW, Lehmann D. Otitis media: viruses, bacteria, biofilms and vaccines. Med J Aust. 2009;191(S9):S44-9. doi: 10.5694/j.1326-5377.2009.tb02926.x. [PubMed: 19883356]. [PubMed Central: PMC7168357].

7. Nevisi F, Bayat M, Hashemi M, Roudbar mohammadi S, Sa'adatmand S. [Comparison of Molecular typing of allergenic fungi Aspergillus fumigatus and Fusarium solani by PCR Colony and PCR methods].J Microb Biotechnol. 2011;2(6):39-46. Persian.

8. Xing-Sheng Y, Yong-Zhi L, Jie-Xin L, Yu-Qing G, Zhang-Huang C, ChongFa Z, et al. Genetic influence on baroreflex sensitivity in normotensive young men. Am J Hypertens. 2010;23(6):655-9. doi: 10.1038/ajh.2010.30. [PubMed: 20300066].

9. Wormald PJ. Treating acute sinusitis. Aust Prescr. 2000;23(2):39-42. doi: 10.18773/austprescr.2000.039. 
10. Wang L, Yokoyama K, Miyaji M, Nishimura K. Mitochondrial cytochrome $\mathrm{b}$ gene analysis of Aspergillus fumigatus and related species. J Clin Microbiol. 2000;38(4):1352-8. doi: 10.1128/JCM.38.4.13521358.2000. [PubMed: 10747106]. [PubMed Central: PMC86444].

11. Brook I. Acute and chronic bacterial sinusitis. Infect Dis Clin North Am. 2007;21(2):427-48. vii. doi: 10.1016/j.idc.2007.02.001. [PubMed: 17561077].

12. Buzina W, Lang-Loidolt D, Braun H, Freudenschuss K, Stammberger H. Development of molecular methods for identification of Schizophyllum commune from clinical samples.J Clin Microbiol. 2001;39(7):23916. doi: 10.1128/JCM.39.7.2391-2396.2001. [PubMed: 11427544]. [PubMed Central: PMC88160].

13. Vernet G. Molecular diagnostics in virology. J Clin Virol. 2004;31(4):239-47. doi:10.1016/j.jcv.2004.06.003. [PubMed: 15494263]. [PubMed Central: PMC7108418].

14. He D, Hao J, Zhang B, Yang Y, Song W, Zhang Y, et al. Pathogenic spectrum of fungal keratitis and specific identification of Fusarium solani. Invest Ophthalmol Vis Sci. 2011;52(5):2804-8. doi: 10.1167/iovs.105977. [PubMed: 21273551].

15. Nowrozi H; Alavi Sh; Kazemi A; Razmpa E; Emami M; Oshaghi M. [Fungus paranasal sinusitis in non- immunocompromised patients in Tehran, Iran (2006-07)].J Gorgan Univ Med Sci. 2012;14(1):129-35. Persian.

16. Naghibzadeh B, Razmpa E, Alavi S, Emami M, Shidfar M, Naghibzadeh $\mathrm{G}$, et al. Prevalence of fungal infection among Iranian patients with chronic sinusitis. Acta Otorhinolaryngol Ital. 2011;31(1):35-8. [PubMed: 21808461]. [PubMed Central: PMC3146333].

17. Biswas SK, Wang L, Yokoyama K, Nishimura K. Molecular analysis of Cryptococcus neoformans mitochondrial cytochrome b gene sequences. J Clin Microbiol. 2003;41(12):5572-6. doi: 10.1128/JCM.41.12.5572-5576.2003. [PubMed: 14662942]. [PubMed Central: PMC308988]. 